\title{
A Two-Stage Data-Driven Multi-Energy Management Considering Demand Response
}

\author{
Pengfei Zhao \\ Department of Electronic and \\ Electrical Engineering \\ University of Bath \\ Bath, United Kingdom \\ P.zhao@bath.ac.uk
}

\author{
Chenghong $\mathbf{G u}^{\dagger}$ \\ Department of Electronic and \\ Electrical Engineering \\ University of Bath \\ Bath, United Kingdom \\ C.Gu@bath.ac.uk
}

\author{
Zhidong Cao \\ The State Key Laboratory of \\ Management and Control for \\ Complex Systems, \\ Institute of Automation, Chinese \\ Academy of Sciences, \\ Beijing, China \\ Zhidong.Cao@ia.ac.cn
}

\begin{abstract}
This paper proposes an innovative two-stage data-driven optimization framework for a multi-energy system. Enormous energy conversion technologies are incorporated in the system to enhance the overall energy utilization efficiency, i.e., combined heat and power, power-to-gas, gas furnace, and ground source heat pump. Furthermore, a demand response program is adopted for stimulating the load shift of customers. Accordingly, both the economic performance and system reliability can be improved. The endogenous solar generation brings about high uncertainty
\end{abstract}

Permission to make digital or hard copies of all or part of this work for personal or classroom use is granted without fee provided that copies are not made or distributed for profit or commercial advantage and that copies bear this notice and the full citation on the first page. Copyrights for components of this work owned by others than ACM must be honored. Abstracting with credit is permitted. To copy otherwise, or republish, to post on servers or to redistribute to lists, requires prior specific permission and/or a fee. Request permissions from Permissions@acm.org.

UbiComp/ISWC '20 Adjunct, September 12-16, 2020, Virtual Event, Mexico (C) 2020 Association for Computing Machinery. ACM ISBN 978-1-4503-8076-8/20/09..\$15.00

https://doi.org/10.1145/3410530.3414587 and variability, which affects the decision making of the system operator. Therefore, a two-stage data-driven distributionally robust optimization (TSDRO) method is utilized to capture the uncertainty. A tractable semidefinite programming reformulation is obtained based on the duality theory. Case studies are implemented to demonstrate the effectiveness of applying the TSDRO on energy management.

\section{CCS CONCEPTS}

- Power system operation - Optimization method

\section{KEYWORDS}

Demand response, Energy hub systems, Multi-energy systems.

\section{ACM Reference Format:}

Pengfei Zhao, Chenghong Gu, Zhidong Cao, Yue Xiang, Xiaohe Yan, and Da Huo. 2020. A Two-Stage Data-Driven Multi-Energy Management Considering Demand Response. In Combining Physical and Data-Driven Knowledge in Ubiquitous Computing, September 13, 2020, Cancun, Mexico.

\section{NOMENCLATURE}


This section presents the description of the sets, parameters and variables, which are used in the mathematical modelling.

A. Sets

\begin{tabular}{|l|l|}
\hline$T$ & Set of time periods. \\
\hline
\end{tabular}

\section{B. Parameters}

\begin{tabular}{|c|c|}
\hline$\eta_{P 2 G}$ & $\begin{array}{l}\text { Conversion efficiency of power-to-gas } \\
\text { (P2G) facility. }\end{array}$ \\
\hline$H H V$ & $\begin{array}{l}\text { Higher heat value of combined heat and } \\
\text { power (CHP). }\end{array}$ \\
\hline$\eta_{e}, \eta_{t h}$ & Electrical and thermal efficiency of CHP. \\
\hline$\eta_{P 2 G}$ & Electrical efficiency of electrolyser. \\
\hline$\eta_{f}, \eta_{r e}$ & $\begin{array}{l}\text { Efficiency of GF and solar power } \\
\text { generation. }\end{array}$ \\
\hline $\mathrm{COP}$ & Coefficient of performance. \\
\hline $\begin{array}{l}\overline{P_{g, C H P}}, \overline{P_{e, H P}}, \\
\overline{P_{g, G F}}\end{array}$ & $\begin{array}{l}\text { Maximum input limit of CHP, ground } \\
\text { source heat pump (GSHP) and gas furnace } \\
\text { (GF). }\end{array}$ \\
\hline$\frac{P_{g, C H P}, P_{e, H P}}{P_{g, G F}}$ & $\begin{array}{l}\text { Minimum input limit of CHP, GSHP and } \\
\text { GF. }\end{array}$ \\
\hline$\overline{\overline{P_{h s}^{c h}}}, P_{h s}^{c h}$ & $\begin{array}{l}\text { Maximum and minimum limit of charging } \\
\text { power of heat storage. }\end{array}$ \\
\hline$\overline{P_{h s}^{d c h}}, \underline{P_{h s}^{d c h}}$ & $\begin{array}{l}\text { Maximum and minimum limit of } \\
\text { discharging power of heat storage. }\end{array}$ \\
\hline$P_{h s}^{s t b}(t)$ & $\begin{array}{l}\text { Standby power loss of heat storage at time } \\
\text { t. }\end{array}$ \\
\hline$E_{h s, \min }, E_{h s, \max }$ & $\begin{array}{l}\text { Minimum and maximum energy for heat } \\
\text { storage. }\end{array}$ \\
\hline$\eta_{h s}^{c h}, \eta_{h s}^{d c h}$ & $\begin{array}{l}\text { Charging and discharging efficiency of } \\
\text { heat storage. }\end{array}$ \\
\hline$\overline{P_{b}^{c h}}, \underline{P_{b}^{c h}}$ & $\begin{array}{l}\text { Maximum and minimum limit of charging } \\
\text { power of battery. }\end{array}$ \\
\hline$\overline{P_{b}^{d c h}}, \underline{P_{b}^{d c h}}$ & $\begin{array}{l}\text { Maximum and minimum limit of } \\
\text { discharging power of battery. }\end{array}$ \\
\hline$E_{b, \min }, E_{b, \max }$ & $\begin{array}{l}\text { Minimum and maximum energy for } \\
\text { battery. }\end{array}$ \\
\hline$\eta_{b}^{c h}, \eta_{b}^{d c h}$ & $\begin{array}{l}\text { Charging and discharging efficiency of } \\
\text { battery. }\end{array}$ \\
\hline$\omega_{f}(t)$ & Solar generation forecast at time $\mathrm{t}$. \\
\hline$\overline{P_{\text {ele }}}, P_{\text {ele }}$ & $\begin{array}{l}\text { Maximum and minimum limit of } \\
\text { electricity purchase. }\end{array}$ \\
\hline$\overline{\overline{P_{\text {gas }}}}, P_{\text {gas }}$ & $\begin{array}{l}\text { Maximum and minimum limit of gas } \\
\text { purchase. }\end{array}$ \\
\hline$\pi_{\text {ele }}(t), \pi_{\text {gas }}(t)$ & Electricity and gas purchase cost at time t. \\
\hline$L_{e}(t), L_{t h}(t)$ & Electricity and heating demand at time t. \\
\hline$D R_{\text {ele,max }}, D R_{t h, \max }$ & $\begin{array}{l}\text { Maximum limit for demand response } \\
\text { coefficient. }\end{array}$ \\
\hline
\end{tabular}

C. Variables

\begin{tabular}{|c|l|}
\hline$E C_{\text {ele }}, E C_{\text {gas }}$ & Energy purchase cost of power and gas. \\
\hline$\omega_{P 2 G}(t), P_{g, P 2 G}(t)$ & Input and output of $\mathrm{P} 2 \mathrm{G}$ electrolyzer. \\
\hline
\end{tabular}

\begin{tabular}{|c|c|}
\hline $\begin{array}{l}P_{g, C H P}(t), P_{e, H P}(t), \\
P_{g, G F}(t)\end{array}$ & Input of CHP, GSHP and GF. \\
\hline $\begin{array}{l}P_{C H P, \text { Eout }}(t), \\
P_{C H P, \text { Hout }}(t), \\
P_{H P, \text { out }}(t), P_{G F, \text { out }}(t)\end{array}$ & Output of CHP, HP and GF. \\
\hline$P_{h s}^{c h}(t), P_{h s}^{d c h}(t)$ & $\begin{array}{l}\text { Charging and discharging power of heat } \\
\text { storage at time } t \text {. }\end{array}$ \\
\hline$u_{h s}^{c h}(t), u_{h s}^{d c h}(t)$ & $\begin{array}{l}\text { Charging and discharging status of heat } \\
\text { storage at time t. }\end{array}$ \\
\hline$P_{b}^{c h}(t), P_{b}^{d c h}(t)$ & $\begin{array}{l}\text { Charging and discharging power of } \\
\text { battery at time t. }\end{array}$ \\
\hline$u_{b}^{c h}(t), u_{b}^{d c h}(t)$ & $\begin{array}{l}\text { Charging and discharging status of } \\
\text { battery at time t. }\end{array}$ \\
\hline$E_{h s}(t), E_{b}(t)$ & $\begin{array}{l}\text { Remaining capacity of heat and battery } \\
\text { storage. }\end{array}$ \\
\hline$\omega_{r}(t)$ & $\begin{array}{l}\text { Scheduled solar power generation at time } \\
\text { t. }\end{array}$ \\
\hline$P_{\text {ele }}(t)$ & Electricity purchase at time t. \\
\hline$P_{\text {gas }}(t)$ & Gas purchase at time t. \\
\hline$v_{e}(t), v_{g}(t)$ & Dispatch factors of electricity and gas. \\
\hline$P_{\text {ele }}^{D R}(t), L_{\text {ele }}^{D R}(t)$ & $\begin{array}{l}\text { Shifted and resulting electricity load } \\
\text { under demand response at time t. }\end{array}$ \\
\hline$P_{t h}^{D R}(t), L_{t h}^{D R}(t)$ & $\begin{array}{l}\text { Shifted and resulting heating load under } \\
\text { demand response at time t. }\end{array}$ \\
\hline
\end{tabular}

\section{INTRODUCTION}

The modern energy systems tend to strengthen the linkage among multi-energy systems (MESs), which significantly aggregates the local generation, distribution and consumption level by end energy customers with new technologies $[1,2]$. This trend requires a basic understanding of the modelling, operation and conversion among all the sub-energy systems. Emerging research efforts are dedicated to resolving the challenges of decarbonization, facilitating renewable penetration and reducing the operation cost based on MESs [3-5].

However, interdependencies and strong couplings have been always the main challenges without an effective solution [6]. Moreover, another limitation of the current research is that the inherent uncertainties cannot be well handled due to the limited data availability, which inevitably affects the system economic performance. Energy hub is a smart local energy management system from the multi-energy perspective, which relies on enormous conversions among multi-energy vectors [7,8]. The energy hub is an interface between local energy producers and consumers with coordination and complementation of multienergy vectors to economically and effectively satisfy loads. To sum up, there are two challenges with respect to multi-energy management: i) the enormous interdependencies and strong couplings among multi-energy vectors are required to be effectively modelled to enhance the overall energy efficiency; and ii) the renewable uncertainty needs an effective optimization framework with mild conservatism and sufficient data availability. 
This paper aims to provide an optimal multi-energy management scheme for MESs. The uncertain renewable variation and fluctuation are handled by a hierarchical two-stage datadriven approach, which enables to determine an initial day-ahead operation scheme based on renewable forecast and take adaptive recourse actions with renewable realization. Cross-vector demand response (DR) is employed to alter the energy users' consumption pattern and thus to reduce the operation cost. To address challenges of modelling energy interdependencies and improper uncertainty treatment, this paper proposes a two-stage data-driven energy hub scheduling considering DR programs, which aims to minimize the daily operation cost. The energy hub is equipped with photovoltaic (PV), energy converters and energy storage systems. The cross-vector DR includes the participation from customers on electricity and heat loads, which helps to reduce the daily operation cost of the energy hub and shift the peak-load. Multiple energy interdependencies and strong energy couplings are considered, including power-to-gas, power-to-heat and heatto-power. The PV uncertainty is captured via a two-stage DRO framework. Moment information is utilized to construct the ambiguity set. The main advantages of this paper are as follows:

- This paper designs a comprehensive model of the multivector system in the form of an energy hub, where the extensive energy conversion is modelled. The proposed model is effective for improving the mutlti-energy utilization efficiency based on conversion technologies.

- Both DR program for heat and electric load are considered to provide additional flexibility for energy hub operators and the grid.

- A two-stage data-driven DRO model is utilized for hedging against PV uncertainty in terms of limited data set and mild robustness. A moment-based ambiguity set is modelled to capture the PV uncertainty. Dual reformulations are made and a semidefinite programming is finally obtained with ensured tractability.

\section{RELATED WORK}

Paper [9] proposes an optimal energy flow model for interconnected energy hubs considering network constraints. The intermittency of renewable generation is handled by a stochastic optimization (SO) based chance-constrained programming. A Cornish-Fisher expansion is utilized to solve the reformulated problem. A distributionally robust optimization (DRO) is applied for energy hub operation considering the multimodality of PV generation [10]. In order to ensure both the voltage security and economic performance, paper [11] designs a volt-var optimization in MES. A reinforcement learning-based data-driven optimization is designed for optimal operation of MESs [12]. The scheduling decisions of electric vehicles and residential appliances are subsequently obtained.

DR is a viable solution for network operators to stimulate energy customers to shift the flexible load profile. Customers are willing to alter their energy consumption patterns in response to

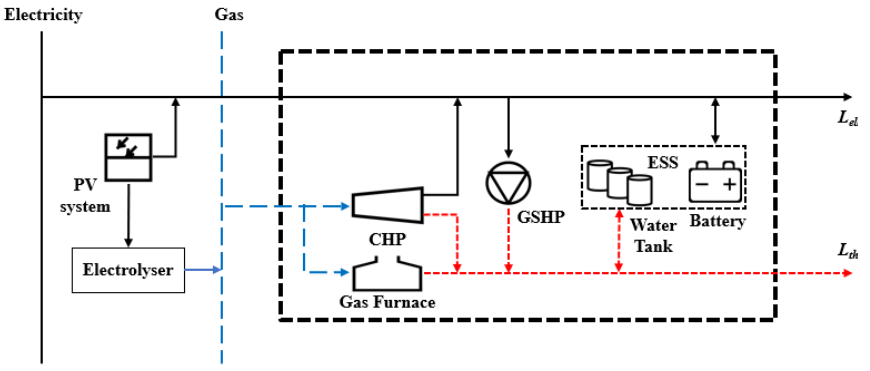

Fig. 1. Overview schematic of the energy hub.

energy price signals. DR in EHS has been investigated in many existing papers. Paper [13] proposes an integrated model for optimal planning of EHS considering DR and renewable energy. A two-stage SO is applied to assess the impacts of uncertainties. The effective DR is embedded into the operational sub-problem. Instead of conventional DR, an integrated DR is proposed in [14] for multiple energy carriers. The interactions between EHSs are modelled as an ordinal potential game. EHSs can participate in DR via switching energy sources during peak demand hours. Paper [15] proposes an optimal probabilistic operation model of EHS with DR. The multi-vector DR contributes to diminishing the operation cost of EHS with customer participation.

To capture the inherent uncertainties in power system operations such as uncertain renewable generation, load demand and contingency events, robust optimization (RO) has been extensively applied. RO makes decisions based on the worst-case scenario, which effectively improves the optimization reliability. Paper [16] proposes a dispatch model of a large-scale hybrid wind/PV/hydro/thermal power system with RO framework. The intermittent power supply is modelled based on the flexible control of robustness. An optimal bidding strategy in a day-ahead microgrid market is proposed in [17]. The intermittency of distributed generation, load variation, and real-time market prices are handled by RO. Paper [18] designs a two-stage RO framework for distribution system reconfiguration, considering load uncertainty. The first stage is to configure the network and the second stage determines the optimal AC power flow. Another classic optimization to capture uncertainties is SO. Compared with RO, SO is another extreme as it assumes the explicit uncertainty distributions [19-21]. Notwithstanding, the solutions are more accurate based on a large number of sample sets and it causes low computational efficiency. Moreover, it is always challenging to obtain sufficient historical data to model real distributions. On the contrary, RO avoids the over-optimistic assumption of uncertainty distributions and provides reliable and computational-efficient solutions.

For DRO, the ambiguity set is constructed by statistical information, such as moment, to restricting possible distributions. Based on more valuable distribution information, research finds that the best estimate of the distribution can be obtained through the statistical fitting. Accordingly, statistical distance information can be added in the ambiguity set and thus the size of the ambiguity set can be controlled. In addition, compared with RO, 
DRO determines expected results over all possible distributions, which are less-conservative. Paper [22] proposes a DRO-based multi-period economic dispatch based on a segeragated linear decision rule. Case studies have demonstrated the effectiveness over the traditional single-period DRO framework. A distributionally robust operation of an electric vehicle aggregator is given in [23], which is proved to outperform RO and SO.

\section{ENERGY HUB SYSTEM MODELLING}

The mathematical modelling of energy hub is presented in this section, including solar PV panels, heat pump (HP), gas furnace (GF), power-to-gas (P2G), combined heat and power (CHP) and a hybrid energy storage system (ESS).

Fig. 1 depicts the proposed energy hub structure, where the power, gas and heat flow are represented by black, blue and red lines. CHP consumes gas and generates power and heat simultaneously. GF converts gas to heat. GSHP enables to convert power to heat with $300 \%$ efficiency. The ESS stores both power and heat based on the surplus energy [24]. The PV system generates solar power via PV panels. However, the generation contains fluctuation due to the unpredicted clouding and weather conditions, which causes PV uncertainty.

\subsection{Objective function}

The energy generation, storage, consumption and extensive energy conversions are realized in proposed energy hub among power, gas and heat. The power and gas purchase from the external markets are the original energy source. The P2G electrolyser converts power to gas with an efficiency of $80 \%$. The ESS is used to store the surplus energy for later usage. This paper adopts a two-stage optimization framework for energy hub scheduling including the day-ahead initial operation scheme based on PV forecast and the real-time adjustive redispatch based on the more accurate PV generation. The objective function shown in (1) to minimize the energy purchase cost. The first-stage objective minimizes the day-ahead operation cost based on PV forecast. And the second-stage objective is to minimize the penalty cost caused by energy purchase deviation ( $E C^{\prime}{ }_{\text {ele }}$ and $E C_{\text {gas }}^{\prime}$ ).

$$
\begin{aligned}
& O b j_{1}=\min \sum_{t \in T} E C_{e l e}+E C_{\text {gas }}, \forall \mathrm{t} \in \mathrm{T} \\
& O b j_{2}=\min \sum_{t \in T} E C^{\prime}{ }_{\text {ele }}+E C_{\text {gas }}^{\prime}, \forall \mathrm{t} \in \mathrm{T} \\
& \text { s.t. (2)-(25) }
\end{aligned}
$$

\subsection{Technical Constraint Formulation}

\subsubsection{Conversion Constraints}

The converted output of P2G, CHP, HP, GF are given in (2)(6), respectively. Constraints (7)-(9) limit the input of CHP, HP and GF.

$$
P_{g, P 2 G}(t)=\eta_{e} \frac{\omega_{P 2 G}(t)}{H H V}, \forall t \in T
$$

$$
\begin{gathered}
P_{C H P, \text { Eout }}(t)=\eta_{e} P_{g, C H P}(t), \forall \mathrm{t} \in \mathrm{T} \\
P_{C H P, H o u t}(t)=\eta_{t h} P_{g, C H P}(t), \forall \mathrm{t} \in \mathrm{T} \\
P_{H P, \text { out }}(t)=C O P P_{e, H P}(t), \forall \mathrm{t} \in \mathrm{T} \\
P_{G F, \text { out }}(t)=\eta_{f} P_{g, G F}(t), \forall \mathrm{t} \in \mathrm{T} \\
\underline{P_{g, C H P}} \leq P_{g, C H P}(t) \leq \overline{P_{g, C H P}}, \forall \mathrm{t} \in \mathrm{T} \\
\frac{P_{e, H P}}{\overline{P_{g, G F}}} \leq P_{e, H P}(t) \leq \overline{P_{e, H P}}, \forall \mathrm{t} \in \mathrm{T} \\
\underline{P_{g, G F}}(t) \leq \overline{P_{g, G F}}, \forall \mathrm{t} \in \mathrm{T}
\end{gathered}
$$

\subsubsection{ESS Constraints}

This paper designs a power-heat hybrid ESS including battery storage and heat storage. The modelling of ESS is given in (10)(14), where $\{\cdot\}$ represents $\{h s, b\}$. Constraints (10) and (11) limit the charging and discharging power and heat. The binary variables $u_{\{\cdot\}}^{c h}(t)$ and $u_{\{\cdot\}}^{d c h}(t)$ are used to ensure the charging and discharging behaviour are not happening in the same time. Constraints (13) and (14) regulate the remaining energy of ESS.

$$
\begin{gathered}
u_{\{\cdot\}}^{c h}(t) \frac{P_{\{\cdot\}}^{c h}}{\overline{d c h}} \leq P_{\{\cdot\}}^{c h}(t) \leq u_{\{\cdot\}}^{c h}(t) \overline{P_{\{\cdot\}}^{c h}}, \forall \mathrm{t} \in \mathrm{T} \\
u_{\{\cdot\}}^{d c h}(t) \frac{P_{\{\cdot\}}^{d c h}}{\frac{u^{c h}}{c h}}(t)+P_{\{\cdot\}}^{d c h}(t) \leq u_{\{\cdot\}}^{d c h}(t) \overline{P_{\{\cdot\}}^{d c h}}, \forall \mathrm{t} \in \mathrm{T} \\
u_{\{\cdot\}}^{d c h}(t) \leq 1, \forall \mathrm{t} \in \mathrm{T} \\
E_{\{\cdot\}}(t)=E_{\{\cdot\}}(t-1)+\sum_{1}^{t} P_{\{\cdot\}}^{c h}(t) \eta_{\{\cdot\}}^{c h}-P_{\{\cdot\}}^{d c h}(t) / \\
\eta_{\{\cdot\}}^{d c h}+P_{\{\cdot\}}^{s t b}(t), \forall \mathrm{t} \in 2 \ldots \mathrm{T} \\
E_{\{\cdot\}, \min } \leq E_{\{\cdot\}}(t) \leq E_{\{\cdot\}, \text { max }}, \forall \mathrm{t} \in \mathrm{T}
\end{gathered}
$$

\subsubsection{Constraints of Energy Purchase}

The power and gas purchase from the external market supplies the energy hub with the modelling given in (15) and (16). Noted that $\{\cdot\}$ denotes $\{$ ele, gas $\}$.

$$
\begin{gathered}
P_{\{\cdot\}} \leq P_{\{\cdot\}}(t) \leq \overline{P_{\{\cdot\}}} \\
E C_{\{\cdot\}}=\pi_{\{\cdot\}}(t) P_{\{\cdot\}}(t), \forall \mathrm{t} \in \mathrm{T}
\end{gathered}
$$

\subsubsection{Energy Balance Conditions}

The balancing condition (coupling matrix) of the energy hub modelling is shown in (17) to guarantee the overall balance between the input and output.

$$
\begin{aligned}
& {\left[\begin{array}{l}
L_{e l e}(t)+P_{b}(t) \\
L_{t h}(t)+P_{h s}(t)
\end{array}\right]=} \\
& {\left[\begin{array}{ccc}
1-v_{e}(t) & \eta_{r e}\left(1-v_{e}(t)\right) & v_{g}(t) \eta_{e}\left(1-v_{e}(t)\right) \\
v_{e}(t) C O P & v_{e}(t) \eta_{r e} C O P & v_{g}(t)\left(\eta_{t h}+\eta_{e} v_{e}(t) C O P+\eta_{f}-v_{g}(t) \eta_{f}\right)
\end{array}\right]} \\
& \times\left[\begin{array}{c}
P_{\text {ele }}(t) \\
\omega_{r}(t)-\omega_{P 2 G}(t) \\
P_{\text {gas }}(t)+P_{g, P 2 G}(t)
\end{array}\right]
\end{aligned}
$$

\subsubsection{Demand Response Conditions}

Energy hub operator implements DR program to encourage the participation of energy customers for gaining economic benefit, i.e., lower energy price at certain time periods is applied which 
stimulates customers to alter their energy consumption profile. The shifted load demand for power and heat are given in (18) and (22) under the maximum limits in (19) and (23). Constraints (20) and (24) regulate the demand deviation owing to the DR program. Equations (21) and (25) guarantees that the overall demand is unchanged.

$$
\begin{gathered}
L_{\text {ele }}^{D R}(t)=L_{\text {ele }}(t)+P_{\text {ele }}^{D R}(t), \forall \mathrm{t} \in \mathrm{T} \\
L_{\text {ele }}^{D R}(t) \leq L_{\text {ele }}^{D R, \text { max }} \\
\left|P_{\text {ele }}^{D R}(t)\right| \leq L_{\text {ele }}(t) D R_{\text {ele }, \text { max }}, \forall \mathrm{t} \in \mathrm{T} \\
\sum_{t=1}^{T} P_{\text {ele }}^{D R}(t)=0, \forall t \in T \\
L_{t h}^{D R}(t)=L_{t h}(t)+P_{t h}^{D R}(t), \forall t \in T \\
L_{t h}^{D R}(t) \leq L_{t h}^{D R, \max } \\
\left|P_{t h}^{D R}(t)\right| \leq L_{t h}(t) D R_{t h, \text { max }}, \forall t \in T \\
\sum_{t=1}^{T} P_{t h}^{D R}(t)=0, \forall t \in T
\end{gathered}
$$

\section{METHODOLOGY}

This section proposes the method for solving the two-stage energy hub operation via DRO model. The abstract formulation and ambiguity set is given firstly, followed by the dual reformulations to ensure the computational tractability. Finally, a semidefinite programming model is obtained.

\subsection{Ambiguity set modelling}

The abstract formulation of the proposed model is given in (26)-(29), where the overall and second-stage objective functions are given in (26) and (28), respectively. Equation (26) is the overall ojective incorporating $O b j_{1}$ and $O b j_{2}$ in (1). And (28) explicitly describes the expected $O b j_{2}$. Equations (27) and (29) represent the constraints in the first and second stages.

$$
\begin{gathered}
\min _{x \in X} c^{\prime} x+\sup _{P f \in D} E_{P}[Q(x, \xi)] \\
\text { s.t. } A x \leq b, \\
Q(x, \xi)=\min _{y} f^{\prime} y \\
\text { s.t. } E x+F y+G \xi \leq h,
\end{gathered}
$$

The data-driven ambiguity set is used to accommodate the statistical information of the uncertain variables. In this paper, the moment information is adopted which reflects the partial distributional information of the PV uncertainty. The fixed mean vector and covariance matrix enable to model a set of possible distributions which share the same moment information. The moment-based data-driven ambiguity set is given in (30).

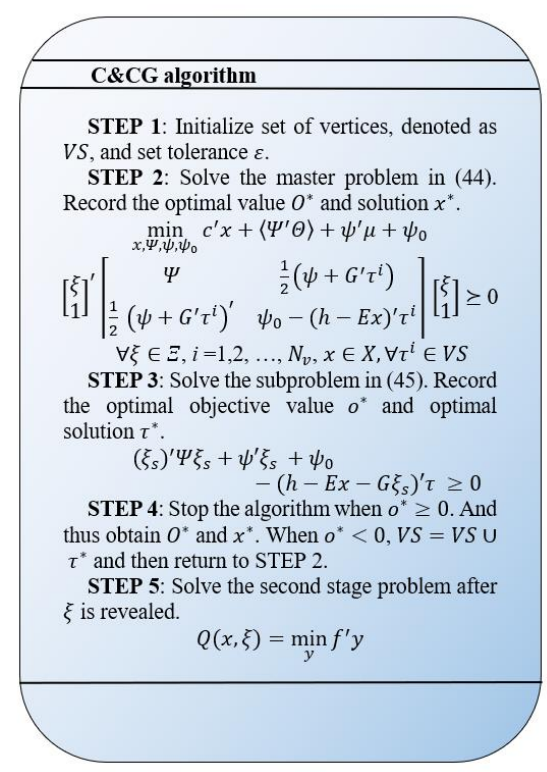

Fig. 2. Flowchart of C\&CG.

$$
D=\left\{\begin{array}{l|c}
f(\xi) & \begin{array}{c}
\mathrm{P}\{\xi\}=1 \\
\mathrm{E}\{\xi\}=\mu \\
\mathrm{E}\left\{\xi(\xi)^{\prime}\right\}=\Sigma+\mu(\mu)^{\prime}
\end{array}
\end{array}\right\}
$$

\subsection{Dual reformulations}

This section proposes two dual reformulations. The first dual reformulation is to merge the two sub-objectives in the first and second stages with 'sup' eliminated. And the second dual reformulation is to transform the infinite variable cardinality to finite cardinality.

The explicit form of $\sup E_{P}[Q(x, \xi)]$ can be rewritten as (31)-(35).

$$
\begin{gathered}
S(x)^{\text {primal }}=\max _{P f \in D_{\xi}} \int_{\Xi} Q(x, \xi) P f(\xi) d \xi \\
\text { s.t. } P f(\xi) \geq 0, \forall \xi \in \Xi \\
\int_{\Xi} P f(\xi) d \xi=1 \\
\int_{\Xi} \xi^{m} P f(\xi) d \xi=\mu_{m}, \mathrm{~m}=1,2, \ldots, \Xi \\
\int_{\Xi} \xi^{m} \xi^{n} P f(\xi) d \xi=\Sigma_{m n}+\mu_{m} \mu_{n}, \mathrm{~m}, \mathrm{n}=1,2, \ldots, \Xi
\end{gathered}
$$

$P f(\xi)$ is the decision variable of (31) which is with infinite cardinality. The dual reformulation enables to transform the infinite-dimensional primal form to the dual form with tractability ensured [25]. The dual reformulation is given in (36) and (38) with dual variables $\psi_{0}, \psi_{j}$ and $\Psi_{j k}$.

$$
\begin{gathered}
S(x)^{\text {dual }}=\min _{\Psi, \psi, \psi_{0}}\left\langle\Psi^{\prime} \Theta\right\rangle+\psi^{\prime} \mu+\psi_{0} \\
\text { s.t. }(\xi)^{\prime} \Psi \xi+\psi^{\prime} \xi+\psi_{0} \geq Q(x, \xi), \forall \xi \in \Xi \\
\min _{x \in X} c^{\prime} x+S(x)^{\text {dual }}
\end{gathered}
$$




\subsection{Semidefinite programming}

Another dual reformulation is required to transform to a closed-form of $Q(x, \xi)$ [26], which is shown in (39) and (40).

$$
\begin{gathered}
\max _{u \in V S} \tau^{\prime}(h-E x-G \xi) \\
V S=\left\{\tau \mid F^{\prime} \tau=f, \tau \leq 0\right\}
\end{gathered}
$$

Equation (41) is the optimal solution of (27) and equations (42) and (43) can be obtained when (41) is substituted by (37).

$$
\begin{gathered}
\exists \tau \in V S: Q(x, \xi)=(h-E x-G \xi)^{\prime} \tau \\
(\xi)^{\prime} \Psi \xi+\psi^{\prime} \xi+\psi_{0} \geq(h-E x-G \xi)^{\prime} \tau \\
\forall \xi \in \Xi, \mathrm{i}=1,2, \ldots, N_{v} \\
(\xi)^{\prime} \Psi \xi+\left(\psi+G^{\prime} \tau^{i}\right)^{\prime} \xi+\psi_{0}-(h-E x) \tau^{i} \geq 0 \\
\forall \xi \in \Xi, \mathrm{i}=1,2, \ldots, N_{v}
\end{gathered}
$$

Thus, equation (44) is the final reformulated objective function to be solved with positive quadractic function in matrix form.

$$
\begin{gathered}
\min _{x, \Psi, \psi, \psi_{0}} c^{\prime} x+\left\langle\Psi^{\prime} \Theta\right\rangle+\psi^{\prime} \mu+\psi_{0} \\
{\left[\begin{array}{l}
\xi \\
1
\end{array}\right]^{\prime}\left[\begin{array}{cc}
\Psi & \frac{1}{2}\left(\psi+G^{\prime} \tau^{i}\right) \\
\frac{1}{2}\left(\psi+G^{\prime} \tau^{i}\right)^{\prime} & \psi_{0}-(h-E x)^{\prime} \tau^{i}
\end{array}\right]\left[\begin{array}{l}
\xi \\
1
\end{array}\right] \geq 0} \\
\forall \xi \in \Xi, \mathrm{i}=1,2, \ldots, N_{v}, x \in X, \forall \tau^{i} \in V S
\end{gathered}
$$

However, the remaining issues is that the vast cardinality of the extreme point set $V S$ causes high computational burden. The proposed column and constraint generation alforithm can solve the large-scale linear models [27], which is given in Fig. 2. And equation (45) presents the sub-problem. The initial set for all the vertices is set in the first step. Then the master and sub problems are solved in turn. At each iteration, the optimal objective value is checked if it is above 0 . If it is not, the set of vertices is updated to incorporate more vertices. When the terminal condition is satisfied, record the optimal value and optimal solution. Then the second-stage problem can be solved based on an expected manner.

$$
\left(\xi_{s}\right)^{\prime} \Psi \xi_{s}+\psi^{\prime} \xi_{s}+\psi_{0}-\left(h-E x-G \xi_{s}\right)^{\prime} \tau
$$

\section{CASE STUDIES}

Case studies are conducted to show the effectiveness of the proposed two-stage data-driven energy hub management with DR. The load shift result based on DR program is given firstly. Then the economic performance and load rescheduling with different pricing schemes are given. The pricing information of real-time pricing (RTP), time-of-use (TOU) and critical peak pricing (CPP) are shown in Fig. 3 and the gas price is fixed at $\$ 0.03 / \mathrm{kWh}[8-10]$. The other technical parameters of the energy hub can be found from the previous literature of the authors $[9,10]$.

\subsection{Impact of Demand Response}

This section proposes the impact of DR programs on system operation. In Figs. 4 and 5, the altered energy patterns are given

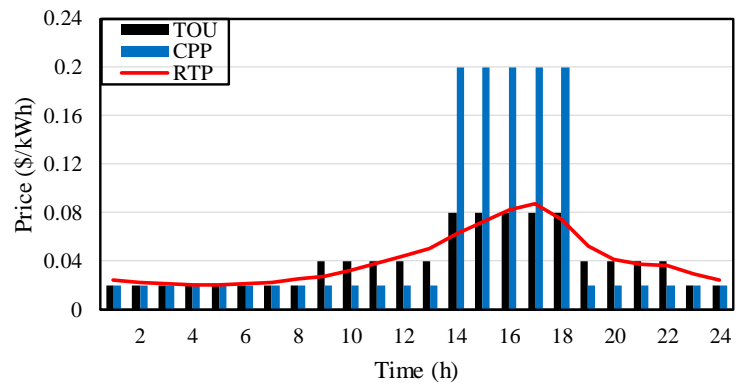

Fig. 3. Pricing mechanisms of TOU, CPP and RTP.

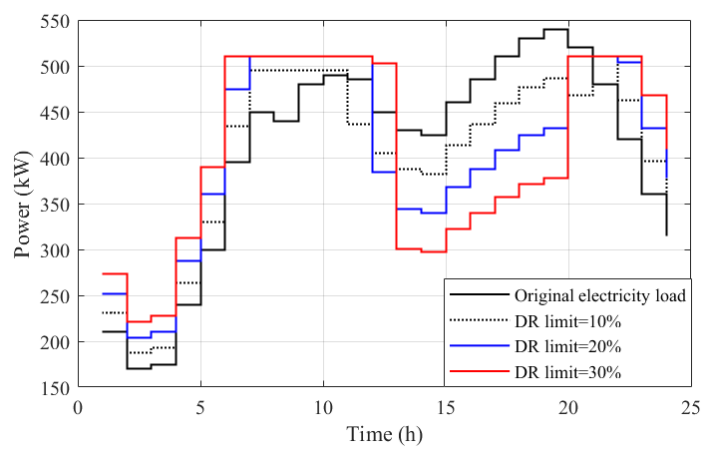

Fig. 4. Electricity load under different DR programs.

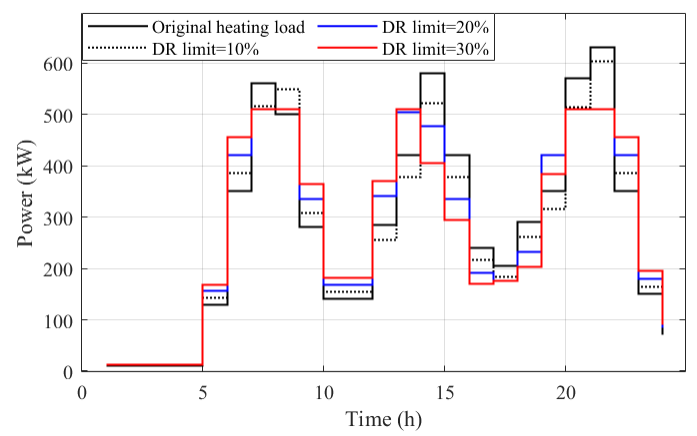

Fig. 5. Heating load under different DR programs.

under different DR programs based on RTP. Fig. 6 shows that the large portion of electricity demand is added during the low-price periods, i.e., from 1:00 to 11:00. While a large amount of electricity load is reduced at the high-price periods, i.e., 13:00 to 20:00. When the DR limit $\left(D R_{\text {ele,max }}\right)$ is increasing, the resulting electricity load consumption pattern is gradually deviating from the original load consumption pattern. For instance, at 19:00, the original electricity load is $540 \mathrm{~kW}$ while it is $486 \mathrm{~kW}, 432 \mathrm{~kW}$ and $378 \mathrm{~kW}$ under the DR limit of $10 \%, 20 \%$ and $30 \%$, respectively. Similarly, Fig. 5 depicts the heating load profile under different DR limits. With the increase of DR limit, more heating load is shifted from the original heating load periods (6:00-10:00, 13:00$16: 00,20: 00-22: 00)$ to the other time periods. 
Table 1: Economic Performance under Different Pricing Schemes

\begin{tabular}{llcc}
\hline \hline Economic result & RTP & TOU & CPP \\
\hline Electricity purchase cost $(\$)$ & 220.80 & 193.50 & 208.24 \\
Gas purchase cost $(\$)$ & 134.92 & 142.74 & 48.82 \\
Total operation cost $(\$)$ & 355.72 & 336.24 & 257.06 \\
\hline \hline
\end{tabular}

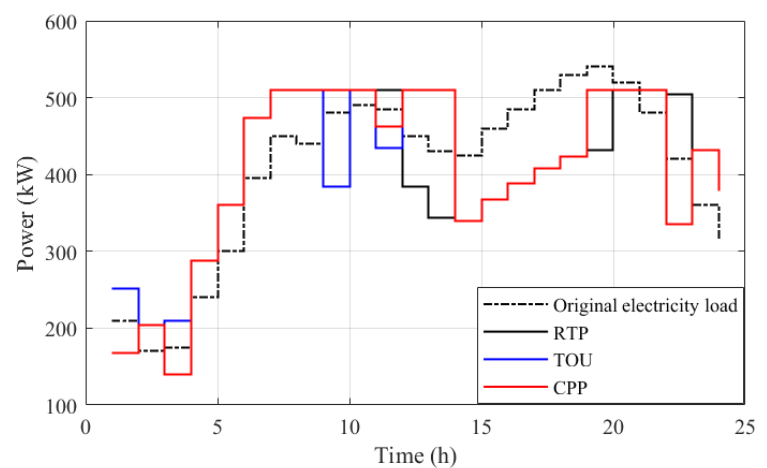

Fig. 6. Electricity load under different DR programs.

\subsection{Application of Different Pricing Mechanisms}

In this section, the comparison is made conducting three different pricing mechanisms, i.e., RTP, TOU and CPP. The economic performance of implementing the three pricing mechanisms is given in TABLE I. Noted that the hybrid uncertainty set is applied. RTP yields the highest electricity purchase cost and total operation cost. The gas purchase cost under TOU scheme is the highest but the total operation cost is $\$ 19$ lower than that under RTP. The reason of the cost difference between RTP and TOU is that the proposed TOU is the approximation of RTP, which is generally lower than RTP. The lower unit purchase cost determines the lower energy purchase cost. In comparison, CPP has a totally different pricing curve than RTP and TOU, i.e., the electricity price during the peak load periods is much higher than those under RTP and TOU. The total operation cost applying CPP is only $\$ 257.06$, which is $72 \%$ and $76 \%$ of the operation cost under RTP and TOU. In addition, the gas purchase cost $(\$ 48.82)$ is much lower than those of RTP and TOU. It is because under DR with RTP, load is extensively shifted to low-price periods. Instead of employing CHP and GF with gas consumption, the shifted electricity profile is more scheduled. In Fig. 6 the altered energy patterns are given under different DR programs among RTP, TOU and CPP. Compared with the original electricity load profile without DR participation, when applying CPP, the consumption profile changes significantly. The load from 14:00 to $18: 00$ is mainly shifted to 6:00-14:00 due to the extremely high electricity tariff at the peak hours.
Table 2: Economic Performance

\begin{tabular}{lccc}
\hline \hline $\begin{array}{l}\text { Economic result } \\
(\$)\end{array}$ & RO & $\begin{array}{c}\text { LDR-based } \\
\text { DRO }\end{array}$ & $\begin{array}{c}\text { The proposed } \\
\text { model }\end{array}$ \\
\hline $\begin{array}{l}\text { First-stage cost } \\
\begin{array}{l}\text { Expected Second- } \\
\text { stage cost }\end{array}\end{array}$ & 444 & 389 & 377 \\
Total cost & 0 & 26 & 21 \\
\hline \hline
\end{tabular}

\subsection{Comparison Analysis}

In order to test the mathematical performance of the proposed two-stage DRO model, a single-stage RO and a DRO model based on linear decision rule are adopted for algorithm comparison. TABLE II shows the economic result of the two stages. A box uncertainty set is utilized for the single-stage RO. Both the firststage cost and total cost are $\$ 444$ without considering the secondstage recourse actions. The proposed moment-based DRO is also compared with a linear decision rule (LDR) based DRO approach, which assumes an affine relationship between the second-stage decisions and uncertain variables [22]. This method conservatively approximates the feasible region of the decision problem, which is computationally efficient. However, the lessconservatism is sacrificed. TABLE II shows that the operation cost of the two stages are \$389 and \$26 via LDR-based DRO. In comparison, the proposed two-stage moment-based DRO reduces the total operation cost by $\$ 17$. To sum up, the proposed model mitigates the over-conservatism by RO and LDR-based DRO.

\section{CONCLUSION}

This paper develops a novel two-stage data-driven optimal operation of energy hub considering cross-vector DR program. The energy hub incorporates multiple energy converters, renewable energy sources and ESS. The uncertain PV generation is effectively modelled via the data-driven ambiguity set with limited distributional information to resolve the limited data availability of using SO. Case studies are conducted to demonstrate the effectiveness of the DR program. This work can benefit both the energy hub operator and customers in terms of saving the operation cost and reducing peak loads.

\section{REFERENCES}

[1] X. Xu, J. Li, Y. Xu, Z. Xu, and C. S. Lai, "A Two-stage Gametheoretic Method for Residential PV Panels Planning Considering Energy Sharing Mechanism," IEEE Transactions on Power Systems, pp. 1-1, 2020, doi 10.1109/TPWRS.2020.2985765.

[2] C. Gu, C. Tang, Y. Xiang, and D. Xie, "Power-to-gas management using robust optimisation in integrated energy systems," Applied Energy, vol. 236, pp. 681-689, 2019/02/15/ 2019, doi: https://doi.org/10.1016/j.apenergy.2018.12.028.

[3] S. Li, H. He, and J. Li, "Big data driven lithium-ion battery modeling method based on SDAE-ELM algorithm and data pre-processing technology," Applied Energy, vol. 242, pp. 
$1259-1273$ 2019/05/15/ 2019 doi: https://doi.org/10.1016/j.apenergy.2019.03.154.

[4] S. Li, J. Li, C. Su, and Q. Yang, "Optimization of BiDirectional V2G Behavior With Active Battery Anti-Aging Scheduling," IEEE Access, vol. 8, pp. 11186-11196, 2020, doi: 10.1109/ACCESS.2020.2964699.

[5] P. Zhao, C. Gu, Z. Hu, X. I. E. D, I. Hernando-Gil, and Y. Shen, "Distributionally Robust Hydrogen Optimization with Ensured Security and Multi-Energy Couplings," IEEE Transactions on Power Systems, pp. 1-1, 2020, doi: 10.1109/TPWRS.2020.3005991.

[6] Y. Shen, C. Gu, Z. Ma, X. Yang, and P. Zhao, "A Two-Stage Resilience Enhancement for Distribution Systems Under Hurricane Attacks," IEEE Systems Journal, pp. 1-9, 2020, doi: 10.1109/JSYST.2020.2997186.

[7] P. Zhao, C. Gu, and D. Huo, "Two-Stage Coordinated Risk Mitigation Strategy for Integrated Electricity and Gas Systems under Malicious False Data Injections," IEEE Transactions on Power Systems, pp. 1-1, 2020, doi: 10.1109/TPWRS.2020.2986455.

[8] P. Zhao, H. Wu, C. Gu, and I. Hernando-Gil, "Optimal home energy management under hybrid photovoltaic-storage uncertainty: a distributionally robust chance-constrained approach," IET Renewable Power Generation, vol. 13, no. 11, pp. 1911-1919, 2019, doi: 10.1049/iet-rpg.2018.6169.

[9] D. Huo, C. Gu, K. Ma, W. Wei, Y. Xiang, and S. L. Blond, "Chance-Constrained Optimization for Multienergy Hub Systems in a Smart City," IEEE Transactions on Industrial Electronics, vol. 66, no. 2, pp. 1402-1412, 2019, doi: 10.1109/TIE.2018.2863197.

[10] P. Zhao, C. Gu, D. Huo, Y. Shen, and I. Hernando-Gil, "TwoStage Distributionally Robust Optimization for Energy Hub Systems," IEEE Transactions on Industrial Informatics, vol. 16, no. 5, pp. 3460-3469, 2020, doi: 10.1109/TII.2019.2938444.

[11] P. Zhao, C. Gu, Y. Xiang, X. Zhang, Y. Shen, and S. Li, "Reactive Power Optimization in Integrated Electricity and Gas Systems," IEEE Systems Journal, pp. 1-11, 2020, doi: 10.1109/JSYST.2020.2992583.

[12] X. Xu, Y. Jia, Y. Xu, Z. Xu, S. Chai, and C. S. Lai, "A Multiagent Reinforcement Learning based Data-driven Method for Home Energy Management," IEEE Transactions on Smart Grid, pp. 1-1, 2020, doi: 10.1109/TSG.2020.2971427.

[13] S. A. Mansouri, A. Ahmarinejad, M. Ansarian, M. S. Javadi, and J. P. S. Catalao, "Stochastic planning and operation of energy hubs considering demand response programs using Benders decomposition approach," International Journal of Electrical Power \& Energy Systems, vol. 120, p. 106030, 2020/09/01/ 2020 https://doi.org/10.1016/j.ijepes.2020.106030.

[14] S. Bahrami and A. Sheikhi, "From Demand Response in Smart Grid Toward Integrated Demand Response in Smart Energy Hub," IEEE Transactions on Smart Grid, vol. 7, no. 2, pp. 650658, 2016, doi: 10.1109/TSG.2015.2464374.

[15] M. Alipour, K. Zare, and M. Abapour, "MINLP Probabilistic Scheduling Model for Demand Response Programs Integrated Energy Hubs," IEEE Transactions on Industrial Informatics, vol. 14, no. 1, pp. 79-88, 2018, doi: 10.1109/TII.2017.2730440.

[16] C. Peng, P. Xie, L. Pan, and R. Yu, "Flexible Robust Optimization Dispatch for Hybrid Wind/Photovoltaic/Hydro/Thermal Power System," IEEE Transactions on Smart Grid, vol. 7, no. 2, pp. 751-762, 2016, doi: 10.1109/TSG.2015.2471102.

[17] G. Liu, Y. Xu, and K. Tomsovic, "Bidding Strategy for Microgrid in Day-Ahead Market Based on Hybrid Stochastic/Robust Optimization," IEEE Transactions on Smart Grid, vol. 7, no. 1, pp. 227-237, 2016, doi: 10.1109/TSG.2015.2476669.
C. Lee, C. Liu, S. Mehrotra, and Z. Bie, "Robust Distribution Network Reconfiguration," IEEE Transactions on Smart Grid, vol. 6, no. 2, pp. 836-842, 2015, doi: 10.1109/TSG.2014.2375160.

[19] T. Zhao, X. Pan, S. Yao, C. Ju, and L. Li, "Strategic Bidding of Hybrid AC/DC Microgrid Embedded Energy Hubs: A TwoStage Chance Constrained Stochastic Programming Approach," IEEE Transactions on Sustainable Energy, vol. 11, no. 1, pp. 116-125, 2020, doi: 10.1109/TSTE.2018.2884997.

[20] A. Najafi, H. Falaghi, J. Contreras, and M. Ramezani, "A Stochastic Bilevel Model for the Energy Hub Manager Problem," IEEE Transactions on Smart Grid, vol. 8, no. 5, pp. 2394-2404, 2017, doi: 10.1109/TSG.2016.2618845.

[21] R. Hemmati, "Stochastic energy investment in off-grid renewable energy hub for autonomous building," IET Renewable Power Generation, vol. 13, no. 12, pp. 2232-2239, 2019, doi: 10.1049/iet-rpg.2018.6254

[22] X. Lu, K. W. Chan, S. Xia, B. Zhou, and X. Luo, "SecurityConstrained Multiperiod Economic Dispatch With Renewable Energy Utilizing Distributionally Robust Optimization," IEEE Transactions on Sustainable Energy, vol. 10, no. 2, pp. 768779, 2019, doi: 10.1109/TSTE.2018.2847419.

[23] X. Lu, K. W. Chan, S. Xia, X. Zhang, G. Wang, and F. Li, "A Model to Mitigate Forecast Uncertainties in Distribution Systems Using the Temporal Flexibility of EVAs," IEEE Transactions on Power Systems, vol. 35, no. 3, pp. 2212-2221, 2020, doi: 10.1109/TPWRS.2019.2951108.

[24] S. Li, H. He, C. Su, and P. Zhao, "Data driven battery modeling and management method with aging phenomenon considered," Applied Energy, vol. 275, p. 115340, 2020/10/01/ 2020, doi: https://doi.org/10.1016/j.apenergy.2020.115340.

[25] E. Delage and Y. Ye, "Distributionally robust optimization under moment uncertainty with application to data-driven problems," Operations research, vol. 58, no. 3, pp. 595-612, 2010.

[26] Y. Chen, W. Wei, F. Liu, and S. Mei, "Distributionally robust hydro-thermal-wind economic dispatch," Applied Energy, vol. 173, pp. 511-519, 2016/07/01/ 2016, doi https://doi.org/10.1016/j.apenergy.2016.04.060.

[27] W. Ben-Ameur and J. Neto, "A constraint generation algorithm for large scale linear programs using multiple-points separation," Mathematical Programming, vol. 107, no. 3, pp. 517-537, 2006/07/01 2006, doi: 10.1007/s10107-005-0694-0. 